\title{
Leserbrief
}

\section{Der Mensch ist keine Maschine}

\author{
In der letzten Ausgabe der "Schmerzmedizin" 2017; 33(4):42-6 berich- \\ tete Manfred Müller über die "Anwendung von Sicherheitsstrategien \\ der Luftfahrt auf die moderne Medizin“. Dazu erhielt die Redaktion \\ folgenden Leserbrief.
}

$\mathrm{H}$ erzlichen Dank für den Artikel! Warum ist die Luftfahrt uns Medizinern hinsichtlich der Risikominimierung um Längen voraus, wie Herr Schürmann in seinem Kommentar richtig bemerkte? So waren die Anfangsbedingungen doch ähnlich: Beide Bereiche arbeiten mit kalkulierten Risiken, sind nicht ungefährlich und entstammen einer strikt hierarchischen Führungskultur. Die Antwort auf diese Frage führt direkt zu den Umständen, die das bislang verhindert haben und weiterhin verhindern werden:

1. In der Luftfahrt hat eine ganze Industrie ein Interesse an der Wahrung ihres sicheren Rufes, sonst steigt der Kunde auf die Bahn um. Die Dienstleistung wird durch ein Ticket der Lufthansa eingekauft und nicht direkt beim Flugkapitän. Das ist in der Medizin anders. Hier ist die Beziehung zwischen Kunden und Dienstleister ungleich persönlicher. Damit wird ein Fehler selten dem Krankenhaus oder einem Krankenhauskonzern angelastet als vielmehr dem einzelnen Arzt. Damit ist die wirtschaftliche Notwendigkeit der Fehlerreduktion in der Medizin weniger priorisiert als in der Luftfahrtindustrie. Auf die Medizin übertragen bedarf es eines übergeordneten Interesses, welches Anreize zur systematischen Fehleranalyse liefert.

2. Piloten können im Simulator üben. Das können wir nicht. Es gibt keine ausgereiften Simulatoren und wird es nie geben, da der Mensch keine standardisierte, technische Maschine ist.

3. Schon erwähnt wurde die Tatsache, dass sich im Cockpit immer zwei Personen befinden. Hier hat die Luftfahrt ein großes Potenzial zur Fehlerreduktion erkannt und umgesetzt, indem die Kommunikation analysiert, standardisiert und modifiziert wurde. Hierarchiestrukturen wurden verändert. Von diesem Wissen kann die Medizin sehr profitieren. Ein Unterschied wird dennoch weiter bestehen: Das Auswahlverfahren der Flugschüler berücksichtigt diese Erkenntnisse, das der Mediziner nicht. Gegebenenfalls erfolgt hierbei sogar eine Negativselektion. Auch unterliegen Mediziner keiner jährlichen Nachschulung und Leistungsüberprüfung.

4. Neben einer zweiten und wohlwollend kontrollierenden Person im Cockpit unterliegen Piloten einer maximalen Überwachung: Voice- und Flightrecorder, Radar-Controller, die sofort Meldung erstatten, wenn man die zugewiesene Höhe missachtet, Computer usw. Es fällt leicht, einen Fehler zuzugeben, wenn sowieso klar ist, dass dieser nicht vertuscht werden kann. Übertragen auf die Medizin würde das die Speicherung von Gesprächen, Vitaldaten und Videoüberwachung bedeuten. Dazu sagte mir ein 747-Pilot, den ich befragte: „Warum eigentlich nicht? Auch im Cockpit werden die Daten nach 30 Minuten überschrieben, es sei denn, ich will, dass sie permanent gespeichert werden." Eindeutig schwieriges Thema!

5. Unterschiede im Umgang mit Fehlern. Eindrücklich beschreibt das der Pilot Martin Bromiley, der 2005 seine Frau bei einer Routineoperation verlor und sich seitdem für den Erkenntnistransfer aus der Fliegerei in die Medizin einsetzt. Er legt Wert darauf, dass die am Tod seiner Frau beteiligten Ärzte weiterarbeiten konnten.

Von den Erkenntnissen aus der Fliegerei kann der Medizinbetrieb nur dann maximal profitieren, wenn die Unterschiede der zwei Bereiche berücksichtigt werden. Konzepte müssen umgearbeitet und mit Augenmaß angepasst werden. Ansätze dazu gab es bereits:

„Dem Chirurgen wird ein schlechter Ausgang in höherem Sinne zur persönlichen Schuld. Tragbar wird diese Belastung durch Gewissenhaftigkeit in der Indikationsstellung, Beherrschung der Technik und ein berechtigtes Selbstbewusstsein. Seine sicherste Stütze aber ist die Wahrhaftigkeit. Der Chirurg, der deutelt, Fehlschläge zu entschuldigen sucht, verstößt gegen das vornehmste Gesetz seiner Zunft“ (Ferdinand Sauerbruch).

Dr. med. Michael Petermeyer, Diez

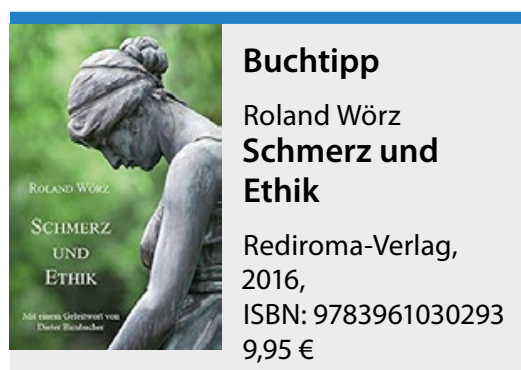

Um es direkt vorweg zu nehmen: Dieses Buch ist ein absolutes Muss für jeden Schmerztherapeuten. Roland Wörz nimmt Sie mit in die Zeitgeschichte der Schmerzmedizin. Sehr schön beschreibt er das schmerztherapeutische Denken und Handeln im zeitgemäßen Zusammenhang mit den großen Philosophen und Ethikern. Bei seinen Beschreibungen verliert er nie den Bezug zum hier und jetzt, auch bei der Umsetzung schwieriger ethischer Fragestellungen. Ein sehr gelungenes und schön zu lesendes Buch. Norbert Schürmann 